\section{In Vitro Study of Esomeprazole Sachet Suspension Administered via Enteral Feeding Tubes}

Administration of drugs through enteral feeding tubes can be a simple way by which infants and children can receive medications, but it introduces concerns about potential blockage of the tube. The risk of feeding tubes becoming blocked is greater with small-diameter feeding tubes and low flush volumes. ${ }^{1}$ Tube blockage is disruptive to patient care, and replacing tubes can be uncomfortable for the children.

Patients with enteral feeding tubes may require acid suppression with proton pump inhibitors (PPIs). Previous studies have assessed the delivery of a single PPI dose through feeding tubes, with delivery of esomeprazole typically being less labour-intensive and more complete than delivery of omeprazole and lansoprazole. ${ }^{1-3}$ However, these studies did not evaluate the potential for blockage with repeated administration of PPIs. This is a real concern, as the tubes may be in place for up to several months.

A new sachet formulation of esomeprazole (Nexium), developed for people who have difficulty swallowing capsules, can be administered by enteral feeding tube. ${ }^{4}$ With the small diameter of the esomeprazole pellets and the formation of a viscous suspension after reconstitution, this formulation was designed to remain stable in solution and to prevent clogging within enteral tubes. ${ }^{4}$

The objective of our in vitro study was to evaluate the potential for clogging (and the resulting change in feeding flow rate) with repeated delivery of 10-mg esomeprazole sachet as suspension through the smalldiameter enteral feeding tubes used at the Children's Hospital of Eastern Ontario and to assess the use of these tubes to deliver the suspension.

Three different types of enteral feeding tubes, each of a different size, were tested: size 6 French Pedi-Tube (Kendall, Mansfield, Massachusetts), size 8.5 French Dawson-Mueller drainage catheter (Cook Canada Inc, Stouffville, Ontario), and size 10 French urological catheter (Bard Canada Inc, Mississauga, Ontario). Four tubes of each type were tested, for a total of 12 tubes. Each tube received a continuous feed of Enfamil A+ formula (Mead Johnson Nutrition, Ottawa, Ontario) at $25 \mathrm{~mL} / \mathrm{h}$ for 7 consecutive days, with an average of $2 \mathrm{~h}$ downtime per day for maintenance and drug administration. For each tube, the formula was delivered by a Kangaroo ePump (Kendall; total of 12 pumps), by means of the pump's compatible $500-\mathrm{mL}$ feed bag set. Each tube was positioned vertically and emptied into its own graduated bottle. For each tube type, 3 of the 4 tubes received the drug suspension; the fourth tube received water as a control.

Every $12 \mathrm{~h}$ the pumps were halted to allow measurement of volumes delivered, replenishment of formula, and administration of the esomeprazole suspensions. With the pumps on hold, the volume that had been fed through each tube was measured (using the respective graduated bottle) and recorded. The predicted volume fed, as displayed on each pump, was also recorded. The exterior of each tube was rinsed with warm water. The feed remaining in the bag sets was discarded, and the feed bags were rinsed with hot water and refilled with $310 \mathrm{~mL}$ of formula. The 10-mg esomeprazole sachets were mixed according to manufacturer's guidelines in $15 \mathrm{~mL}$ sterile water. ${ }^{5}$ The tubes were disconnected from the feed lines one at a time, and the drug suspension or placebo was delivered with a $30-\mathrm{mL}$ syringe. The drug infusion time was the same for each tube ( 4 or $5 \mathrm{~s}$ ). The additional flush of $15 \mathrm{~mL}$ advised by the manufacturer's guidelines was not used, so as to simulate drug administration for a patient with volume restriction. Each tube received a 5-mL flush with sterile water before and after delivery of drug or placebo. After drug administration, the tube was reconnected and the pump restarted. The stop, start, and drug administration times were recorded individually for each pump or tube.

Blockage of the 12 enteral feeding tubes was assessed by measurement of the feed flow rate. Average flow rates were calculated according to the volume delivered for a $12-\mathrm{h}$ period. A change in the calculated feed flow rate was considered significant if it was outside the $\pm 10 \%$ operating range. ${ }^{6}$ The pumps had the capacity to sound an alert when a blockage occurred. If this happened, the tube was removed and was not replaced. The bag set was restarted and run for a short period (30 min) to confirm that the blockage was in the tube and not the bag set.

On the sixth day of continuous feeding with twice-daily drug administration, one complete block occurred immediately after administration of the 11th dose of esomeprazole into 1 of the 3 size 6 French tubes receiving the drug suspension. There was no observable change in the feed flow rate leading up to the blockage in this tube. The only indication of a potential blockage occurred during the final drug administration, when it was more difficult than usual (i.e., more pressure on the syringe was required) to administer the drug through the tube. No blockages occurred in any of the other tubes, and there were no observable changes in feed rates in any of the 3 tube types tested.

Administration of the viscous suspension through the size 6 French tubes required significant pressure on the syringe. Using the dual-port design on these tubes to deliver the drug suspension without detaching the tube resulted in backflow of drug into the feed bag set. When the tube was detached from the bag set to prevent backflow during drug administration, minor leaks from the dual port occurred.

On the basis of the data from this study, we conclude that the potential for blockage exists when esomeprazole sachet as suspension is delivered through size 6 French feeding tubes. However, there were no changes in feed flow rates until complete failure (blockage) of the feeding tube occurred. The other tubes tested showed no signs of blockage or changes in feed flow rate.

\section{References}

1. Messaouik D, Sautou-Miranda V, Bagel-Boithias S, Chopineau J. Comparative study and optimisation of the administration mode of three proton pump inhibitors by nasogastric tube. Int J Pharm 2005;299 (1-2):65-72.

2. White CM, Kalus JS, Quercia R, Fortier C, Piotrowski A, Roach A, et al. Delivery of esomeprazole magnesium enteric-coated pellets through small caliber and standard nasogastric tubes and gastrostomy tubes in vitro. Am J Health Syst Pharm 2002;59(21):2085-2088.

3. Dunn A, White CM, Reddy P, Quercia RA, Chow MSS. Delivery of omeprazole and lansoprazole granules through nasogastric tube in vitro. Am J Health Syst Pharm 1999;56(22):2327-2330.

4. Bladh N, Blychert E, Johansson K, Backlund A, Lundin C, Niazi M, et al. A new esomeprazole packet (sachet) formulation for suspension: in vitro characteristics and comparative pharmacokinetics versus intact capsules/tablets in healthy volunteers. Clin Ther 2007;29(4):640-649. 
5. Nexium [product monograph]. Mississauga (ON): AstraZeneca Canada Inc; 2008 Jun 16.

6. Kendall Kangaroo ePump enteral feeding pump operation and service manual package insert. Mansfield (MA): Tyco Healthcare Group LP; 2007.

\section{Paul Stewart, BScEng}

Natalie Dayneka, BScPhm, PharmD, FCSHP

Lieutenant-Commander Sylvain Grenier, CD, BScPharm, PharmD

Carolyn Stewart, BSP

Lise Beadow, RN

Gerry Joseph

David Mack, MD, FRCPC

Régis Vaillancourt, BPharm, PharmD, FCSHP

Children's Hospital of Eastern Ontario

Ottawa, Ontario

Sylvain Grenier is also affiliated with Canadian Forces Health Services Center Ottawa in Ottawa, Ontario. David Mack is also affiliated with the Department of Pediatrics, University of Ottawa in Ottawa, Ontario.

\section{Acknowledgements}

We thank Covidien for providing all of the pumps and bag sets and Patricia L'Abbé at Covidien for her assistance. Cook Inc provided the Dawson-Mueller drainage catheters. AstraZeneca provided the Nexium sachets and an unrestricted research grant.

\section{ON THE FRONT COVER}

\section{St Mary's Hospital, Sechelt, British Columbia}

The beautiful Lower Sunshine Coast is located on the mainland of British

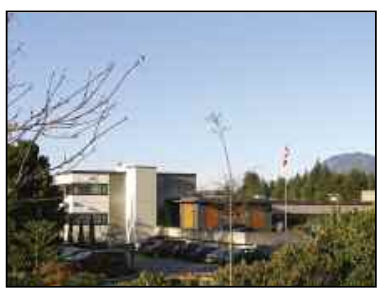
Columbia just a few miles north of Vancouver, but it is accessible only by a 45minute ferry ride. St Mary's Hospital, depicted in this issue's cover photograph, is the community hospital for the entire Lower Coast. The hospital serves about 30000 people in small communities ranging from Gibsons (home of the TV series The Beachcombers) at the south end up to Egmont (home of the famous Skookumchuck Rapids) about $100 \mathrm{~km}$ to the north. The population almost doubles during the summer months with visitors coming to enjoy the many activities available.

St Mary's hospital has 47 beds (intenstive care, medical-surgical, obstetrics, mental health), 2 operating theatres, a 24-hour emergency department, an extended care department, and a very busy ambulatory care and chemotherapy clinic. The Pharmacy Department, staffed by 2 FTE pharmacists and 2.5 FTE technicians, has active clinical (inpatient and outpatient) and chemotherapy programs in addition to a busy dispensary. The department has also implemented a successful medication reconciliation program for both inpatient and pre-operative admissions.

The cover photograph, looking north up Sechelt Inlet, was taken with an Olympus SP-500 UZ camera by Ann Beardsell, Pharmacy Manager at St Mary’s Hospital.

CJHP would be pleased to consider photographs featuring rural hospitals and their pharmacy departments or residential care facilities taken by CSHP members for use on the front cover of the journal. If you would like to submit a photograph, please send an electronic copy (minimum resolution $300 \mathrm{dpi}$ ) to Sonya Heggart at sheggart@cshp.ca. 